\title{
Finite Element Analysis of the Dynamic Response of the Maxillary Molar under Impact Loading
}

\author{
Hai-Tao XIN ${ }^{1,2,3, *}$, Yu-Lu WU1,2,3, Kai LI ${ }^{1,2,3}$, Xiao-Ou DIAO ${ }^{1,2,3}$, Fan \\ FENG $^{1,2,3}$, and Chen-Yun DOU ${ }^{1,2,3}$
}

1State Key Laboratory of Military Stomatology, Department of Prosthodontics, School of Stomatology, The Fourth Military Medical University, Xi'an 710032, China; 2National Clinical Research Center for Oral Diseases, Department of Prosthodontics, School of Stomatology, The Fourth Military Medical

University, Xi'an 710032, China; 3Shannxi Key Laboratory of Stomatology, Department of Prosthodontics, School of Stomatology, The Fourth Military Medical University, Xi'an 710032, China

*xhthmj@126.com, 331617386@qq.com, 54918808@qq.com, 923612930@qq.com, 191837124@qq.com, Rhh-123@163.com.

Keywords: Maxillary molar, Periodontal ligament, Vibration, Dynamic analysis, Response.

\begin{abstract}
The dynamic response of a maxillary molar with periodontal ligament (PDL) was investigated to explore the dynamic behavior of the tooth. Three-dimensional (3D) finite element model of a maxillary molar with periodontium was constructed using CT image-reconstruction. The modal vibration of the maxillary molar in the periodontal system was performed at first, and then the dynamic response of the tooth under an impact loading was calculated and compared with the results of the different periodontal attachments in order to assess the condition of the periodontium. The first four modes were dominant in the modal vibration. The resonant frequency of the maxillary molar under the impact loading was close to the frequency of the second mode in the modal analysis. The resonant frequency decreased gradually with the loss of alveolar bone, while the resonant amplitude increased nonlinearly. The resonant frequency and amplitude of the maxillary molar can be used to describe the loss of alveolar bone and the condition of periodontium.
\end{abstract}

\section{Introduction}

The periodontal ligament (PDL) is a thin soft tissue made up of thousands of fibers which fasten tooth cementum to the bony socket and provide attachment of a tooth to the adjacent alveolar bone to support the tooth. Biomechanical information on the PDL condition is very important in clinical diagnosis, treatment planning and prognosis management $[1,2]$. Many methods are used to evaluate the PDL condition, for example radiographic examination, masticatory pressure and tooth mobility. The dynamic behavior of a tooth in the periodontal system reflects the important parameters for assessing the periodontal tissue condition: the damaged condition of periodontium and the level of periodontal attachment [3, 4]. The dynamic response of a tooth with the PDL is strongly related to the application force and the excitation direction when the vibrational properties of the tooth is used to describe the condition of the periodontium [5]. Transient dynamic analysis is a technique used to determine the dynamic response of a structure under the action of any general time-dependent loads $[6,7]$. The mechanism by which the strain energy is gradually converted to another energy form is known as damping. Some investigations focus on the calculation or measuring of the natural frequency and the damping of a tooth subjected to an external excitation [1,3,4] in vivo and in vitro. But the dynamic behavior of a molar excited by an impact force has not been well explored. In order to investigate the dynamic behavior of a maxillary molar and the relation between the resonant frequency of the tooth and the level of periodontal attachment, dynamic finite element analysis of the maxillary molar in the periodontal system under an impact loading was performed in this study to assess the condition of periodontium. 


\section{Methods}

\section{Finite Element Model}

To construct a 3D geometric model of a maxillary molar and the PDL, CT image-reconstruction technique was used in this study provided by the 3D-DOCTOR software (3D-DOCTOR Version3.5, Able Software Corp, Lexington, MA, USA) at first. The procedure to build the 3D model was referred to our previous study [8]. Then to rebuild the PDL of the maxillary molar, the meshed surface of the tooth root where the PDL attached was selected and expanded outwards to form the PDL tissue based on the thickness of the PDL. Finally, the PDL structure was meshed correspondingly with the maxillary molar. The 3D finite element model of the maxillary molar and the PDL was set up for further analysis (Figure 1). In this model, the length of the tooth was $19.3 \mathrm{~mm}$, the crown was $7.1 \mathrm{~mm}$ and the root was $12.2 \mathrm{~mm}$. The thickness of the PDL was 0.25mm[9]. In order to simulate periodontal attachment loss in vivo, the height of periodontal attachment was defined to describe the condition of alveolar bone loss and the level of periodontal attachment. A series of models was used in the analysis, with a decrement of $1.0 \mathrm{~mm}$ each for the height of periodontal attachment from the anatomic boundary between the crown and the root of the tooth. The mechanical properties for enamel, dentin and the PDL were taken from the references $[6,10]$ (Table $1)$.

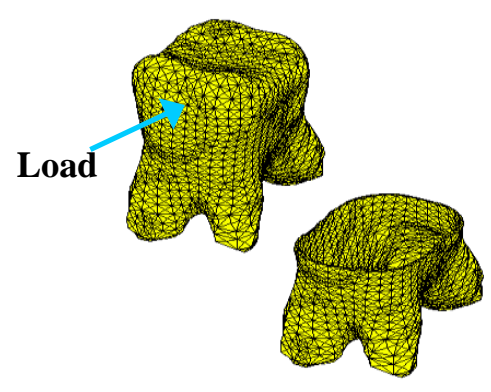

Figure 1. The model of the maxillary molar and PDL

Table 1. Mechanical properties used in the current study

\begin{tabular}{|lccc|}
\hline Material & Young's modulus $(\mathrm{GPa})$ & Poisson's ratio & Density $\left(\mathrm{g} / \mathrm{cm}^{3}\right)$ \\
\hline Enamel & 77.9 & 0.33 & 3.0 \\
Dentin & 16.6 & 0.31 & 2.2 \\
PDL & 0.05 & 0.45 & 1.1 \\
\hline
\end{tabular}

\section{Numerical Approach}

The tooth, the PDL and the alveolar bone were assumed to be a stretch buffer viscoelastic system in this study [11-13] to describe the dynamic property of the maxillary molar in the periodontal system. The alveolar bone was considered immovable, the restrictions of displacement were applied on the exterior surface of the PDL in three-directions $[14,15]$.

To validate the model, the modal analysis of the maxillary molar in the periodontal system was performed at first using MSC software MARC-FEA. The first four mode shapes and frequencies were selected to describe vibration characteristics. Then the acceleration of the tooth under impact loading was calculated and converted into the response by the FFT analyser to describe dynamic property of the tooth. A sinusoidal force (Figure 2) with a peak of $500 \mathrm{~N}$ [9] represented masticatory load, a rise time of $2 \mathrm{~ms}$, and a total duration of $4 \mathrm{~ms}[6,9]$, was chosen and imposed on the central point in buccal surface of the dental crown in the buccal-lingal direction (Figure 1). 


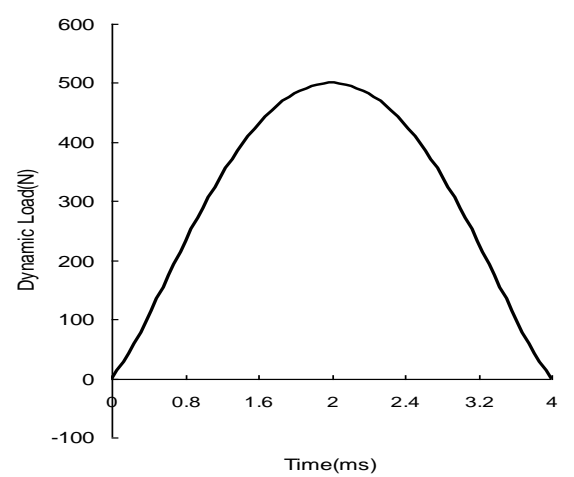

Figure 2. The loading function on the tooth

\section{Result}

\section{The Modal Vibration Analysis of the Maxillary Molar}

The modal analysis of a tooth in the periodontal system is the basis of the tooth dynamics. To explore the vibration characteristic of the maxillary molar in a healthy periodontal condition, in this study, the model of the maxillary molar was attached with the PDL at an ideal level of periodontal attachment on the root. The frequencies and shapes of the first four modes were selected. The results show that the frequency of the first mode was $931 \mathrm{~Hz}$, and the shape was the swing in the mesial-distal direction. The second mode was $1048 \mathrm{~Hz}$, and the shape was in the buccal-lingal direction. The third mode was $1347 \mathrm{~Hz}$, and the shape was the rotation about the longitudinal axis of the tooth. The fourth mode was $1737 \mathrm{~Hz}$, and the shape was in the longitudinal axis of the tooth (Figure 3).

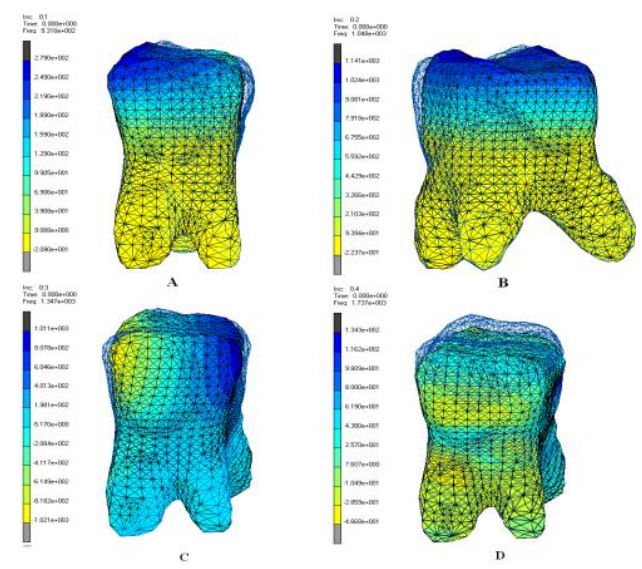

Figure 3. The frequencies and shapes of the first fourth modes. The panel A refers to the first mode. The panel $\mathrm{B}$ refers to the second mode. The panel $\mathrm{C}$ refers to the third mode. The panel $\mathrm{D}$ refers to the fourth mode

\section{The Resonant Frequency of the Maxillary Molar}

The impact acceleration of the maxillary molar in a healthy periodontal condition was calculated under impact loading (Figure 4). The time-domain spectrum of the response result was transformed into a frequency domain spectrum by the FFT analyser. And the resonance frequency was then obtained to describe the resonant characteristics of the maxillary molar. 


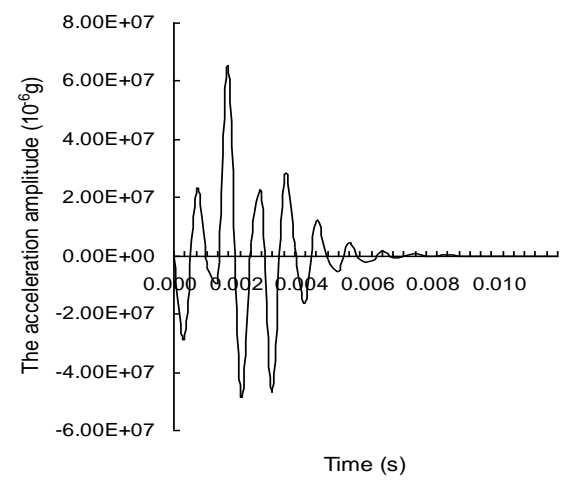

Figure 4. Dynamic response of the maxillary molar

The result shown that the resonant frequency of the maxillary molar under impact loading was $1047 \mathrm{~Hz}$ (Figure 5). The value was close to the frequency $(1048 \mathrm{~Hz})$ of the second mode in the modal analysis above. And the shape of the second mode was in the buccal-lingal way which was the direction also the impact loading in.

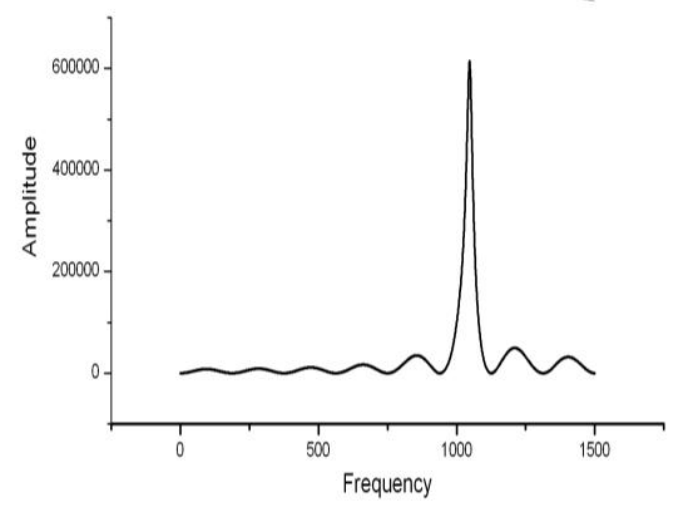

Figure 5. Resonant frequency of the maxillary molar

\section{The Relation between the Attachment of the PDL and the Dynamic Response}

\section{The Relation between the Attachment of the PDL and the Resonant Frequency}

The resonant frequency has relations with the periodontal tissue condition[4, 8]. In order to investigate the relationship between the resonant frequency and the alveolar bone loss, the height of periodontal attachment was defined to describe the condition of alveolar bone loss and the level of periodontal attachment. A series of virtual models was used in the analysis, with a decrement of $1.0 \mathrm{~mm}$ each for the height of periodontal attachment from the anatomic boundary between the crown and the root of the tooth. The results (Figure 6) shown that the resonant frequencies decreased gradually with the loss of ligament attachment under the same impact loading. 


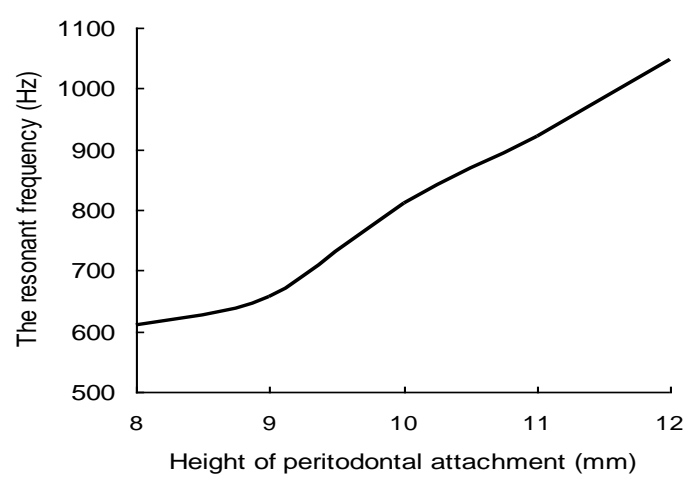

Figure 6. Effect of the height of periodontal attachment on the resonant frequency

From the results above it can be seen that the height of periodontal attachment is correlative with the resonant frequency, and the resonant frequency of the maxillary molar can be used to describe the loss of alveolar bone and the level of periodontal attachment.

\section{The Relation between the Attachment of the PDL and the Resonant Amplitude}

The resonant amplitude of the maxillary molar increased nonlinearly with the loss of alveolar bone under the impact loading (Figure 7). When the height of periodontal attachment was higher than 10 $\mathrm{mm}$, the resonant amplitude increased gradually, but when the height of periodontal attachment decreased further, the resonant amplitude increased quickly. This indicates that the resonant amplitude can be used to describe the mobility of the maxillary molar.

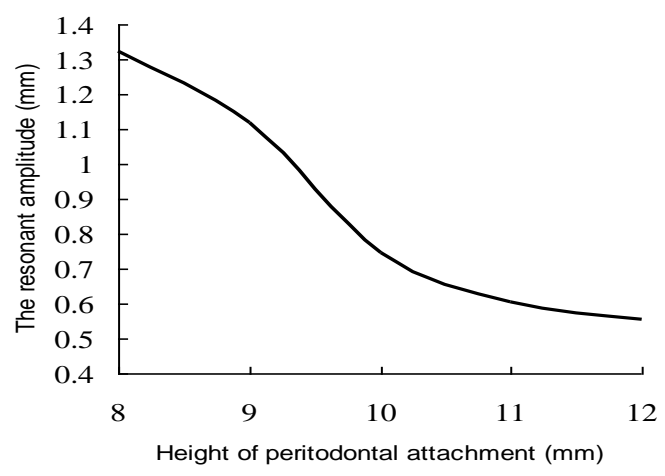

Figure7. Effect of the height of periodontal attachment on the resonant amplitude

\section{Discussion}

The condition of the periodontal tissue is very important in the diagnosis and treatment planning [16, 17]. Many methods are used to evaluate the condition of the PDL [18, 19], but most of them focus on the morphologic change of tissue or measurement in static state for example radiographic examination to assess the PDL condition. The support of a tooth is provided by the combined action of the PDL and the alveolar bone, and the PDL is a thin viscoelastic tissue made up of thousands of fibers to support the tooth $[20,21]$. The dynamic property of a tooth is proved to be a valid way to evaluate the condition of periodontal tissues $[15,22]$. Many investigations concentrate on measuring vibration frequency of a tooth in vivo and in vitro [23, 24]. But the dynamic behavior of a tooth has not been well explored, especially to a molar excited by an impact force. In this study, the modal analysis and dynamic response are performed to investigate the dynamic behavior of a maxillary molar. It is found from the results that the first four modes in the modal vibration are dominant whose shapes are in substantial alignment with the directions of the tooth mobility [8]. The resonant 
frequency $(1047 \mathrm{~Hz})$ of the maxillary molar under impact loading is close to the frequency $(1048 \mathrm{~Hz})$ of the second mode in the modal analysis. And the shape of the second mode is in the buccal-lingal direction as is the impact. The result is also near to the frequency $1190 \pm 130 \mathrm{~Hz}$ in the buccal-lingal direction measured by Huang et al [25] taking into account the effect of measurement error. This study confirms the feasibility of measuring a tooth mobility in clinical application. And it should be appropriate to use dynamic response to describe the PDL condition [26] under a certain practical excitation.

Periodontitis results in the loss of alveolar bone and the recession of the periodontal attachment. According to the literature concerning tooth mobility, the vibration frequency of a tooth in the periodontal system is related with the level of periodontal attachment $[4,8]$. The present study shows that the resonant frequency of the maxillary molar decreases gradually with the loss of alveolar bone under impact loading, while the resonant amplitude increases nonlinearly. This result demonstrates that the resonant frequency and amplitude of the maxillary molar can be used to describe the loss of alveolar bone and the mobility of the maxillary molar.

For measuring the resonant frequency of the tooth in clinical application, the impulse force applied on the tooth must be assigned at first. When a suitable force is used in vivo testing, the resonant frequency and amplitude of the maxillary molar measured in vivo can be used to describe the loss of alveolar bone and evaluate the periodontal condition.

\section{Acknowledgements}

This study was supported by National Natural Science Foundation of China (Contract no. 11672327).

\section{References}

1. Kojima Y, and Fukui H, Calculation of natural frequencies of teeth supported with the periodontal ligament, Dent Mater J 2007; 26 (2): 254-259.

2. Cronau M, Ihlow D, Kubein-Meesenburg D and Fanghänel J, Biomechanical features of the periodontium: An experimental pilot study in vivo, Am J Orthod Dentofacial Orthop 2006; 129 (5): $13-21$.

3. Shen LK, Huang HM, and Yu JJ, et al, Sung-Chih Hsieh, Effects of periodontal bone loss on the natural frequency of the human canine: a three-dimensional finite element analysis.2009; J Dent Sci 4(2): 81-86.

4. Lee SY, Huang HM, Lin CY and Shih YH, In vivo and in vitro natural frequency analysis of periodontal conditions: an innovative method, J Periodontol 2000; 71 (4): 632-40.

5. Melsen B, Cattaneo PM, Dalstra M and Kraft DC, The Importance of Force Levels in Relation to Tooth Movement, Semin Orthod 2007; 13 (4): 220-233.

6. Huang HM, Ou KL, and Wang WN et al, Dynamic Finite Element Analysis of the Human Maxillary Incisor Under Impact Loading in Various Directions. J Endod 2005; 31(10): 723-727.

7. Corte's F and Elejabarrieta MJ, Computational methods for complex eigenproblems in finite element analysis of structural systems with viscoelastic damping treatments, Comput. Methods Appl. Mech. Engrg 2006; 195(47): 6448-6462.

8. Haitao Xin, Yulong Li, Lingcheng Zhao and Weiguo Guo, Nonlinear finite element analysis of the vibration characteristics of the maxillary central incisor related to periodontal attachment, Med Biol Eng Comput 2009; 47(11): 1189-1195.

9. Pi Xin. Dental Anatomy Physiology. Beijing, China: People's Medical Publishing House; 2007.

10. Natali AN, Pavan PG, Scarpa C, Numerical analysis of tooth mobility: formulation of a 
non-linear constitutive law for the periodontal ligament, Dent Mater 2004; 20 (7): 623-629.

11. Tanaka E, Inubushi T, Takahashi K and Shirakura M, Dynamic shear properties of the porcine molar periodontal ligament, J Biomech 2007; 40 (7): 1477-1483.

12. Pietrzak G, Curnier A, and Botsis J, et al, A nonliear elastic model of the periodontal ligament and its numerical calibration for the study of the tooth mobility. Comput Method Biomech Biomed Eng 2002; 5(2): 91-101.

13. Ziegler A, Keilig L, Kawarizadeh A and Jäger A, Numerical simulation of the biomechanical behaviour of multi-rooted teeth, Eur J Orthodont 2005; 27 (5): 333-339.

14. Huang H-M, Tsai C-Y, Lee H-F and Lin C-T, Damping effects on the response of maxillary incisor subjected to a traumatic impact force: A nonlinear finite element analysis, J Dent 2005; 27 (6): $1-8$.

15. Yamane M, Yamaoka M, Hayashi M and Furutoyo I, Measuring tooth mobility with a no-contact vibration device, J Periodont Res 2008; 43 (1): 84-89.

16. Noyes DH and Solt CW, Measurement of mechanical mobility of human incisors with sinusoidal forces, J Biomech 1973; 6 (5): 439-442.

17. Yang SM, Shin SY, and Kye SB, Relationship between implant stability measured by resonance frequency analysis (RFA) and bone loss during early healing period. Oral Surg Oral Med Oral Pathol Oral Radiol Endod, 2008; 105(2): 12-19.

18. Robertson LT, Levy JH, and Petrisor D, Vibration perception thresholds of human maxillary and mandibular central incisors. Arch Oral Biol 2003; 48(4): 309-316.

19. Lin SL, Lee S-Y, Lee LY and Chiu W-T, Viberation analysis of mandible trauma: experimental and numerical approaches, Med Biol Eng Comput 2006; 44(9): 785-792.

20. Slomka N, Vardimon AD, Gefen A and Pilo R, Time-related PDL: viscoelastic response during initial orthodontic tooth movement of a tooth with functioning interproximal contact-A mathematical model, J Biomech 2008; 41 (9): 1871-1877.

21. Sanctuary CS, Wiskott HWA, and Justiz J et al, In vitro time-dependent response of periodontal ligament to mechanical loading. J. Appl Physiol 2005; 99 (6): 2369-2378.

22. Schliephake H, Sewing A, and Aref A, Resonance frequency measurements of implant stability in the dog mandible: experimental comparison with histomorphometric data. Int. J. Oral Maxillofac. Surg 2006; 35(5): 941-946.

23. Castellini P, Scalise L, and Revel GM, Vibration measurements for diagnosis of structural defects on human teeth. Meas 2000; 27(1): 29 -42.

24. Nishimura $M$, Chiba $M$, and Ohashi $T$ et al, Periodontal tissue activation by vibration: Intermittent stimulation by resonance vibration accelerates experimental tooth movement in rats. Am J Orthod Dentofacial Orthop 2008; 133 (4): 572-583.

25. Huang HM, Yeh CY, and Lee SY et al, Factors influencing the dynamic behaviour of human teeth. Med Biol Eng Comput 2001; 39 (4): 176-181.

26. Bergomi M, Cugnoni $\mathrm{J}$, and Botsis $\mathrm{J}$, The role of the fluid phase in the viscous response of bovine periodontal ligament. J Biomech 2010; 43(6): 1146-1152. 